\title{
Nonlinear optical imaging of defects in cubic silicon carbide epilayers
}

SUBJECT AREAS:

MULTIPHOTON

MICROSCOPY

IMAGING TECHNIQUES

Received

20 March 2014

Accepted

23 May 2014

Published

11 June 2014

Correspondence and requests for materials should be addressed to

G.A.S. (stanciu@ physics.pub.ro)

\author{
Radu Hristu, Stefan G. Stanciu, Denis E. Tranca, Alecs Matei \& George A. Stanciu
}

Center for Microscopy-Microanalysis and Information Processing, University Politehnica of Bucharest, 313 Splaiul Independentei, 060042 Bucharest, Romania.

Silicon carbide is one of the most promising materials for power electronic devices capable of operating at extreme conditions. The widespread application of silicon carbide power devices is however limited by the presence of structural defects in silicon carbide epilayers. Our experiment demonstrates that optical second harmonic generation imaging represents a viable solution for characterizing structural defects such as stacking faults, dislocations and double positioning boundaries in cubic silicon carbide layers. X-ray diffraction and optical second harmonic rotational anisotropy were used to confirm the growth of the cubic polytype, atomic force microscopy was used to support the identification of silicon carbide defects based on their distinct shape, while second harmonic generation microscopy revealed the detailed structure of the defects. Our results show that this fast and noninvasive investigation method can identify defects which appear during the crystal growth and can be used to certify areas within the silicon carbide epilayer that have optimal quality.

r he need for new generation high power electronic devices capable of operating at extreme conditions has made silicon carbide $(\mathrm{SiC})$ extensively investigated over the past two decades because its properties propose it as an extremely viable solution ${ }^{1}$. These properties include a higher breakdown field than that of silicon which permits much smaller drift regions, a higher thermal conductivity, which allows better heat dissipation and a wide band gap energy which enables higher operating temperatures, making SiC suitable for high-temperature, high-frequency and high-power electronic devices. In addition, its strong bonding and large optical phonon energies make $\mathrm{SiC}$ attractive also for applications in nonlinear optics, especially for ones that deal with short wavelength high optical power devices or high operating temperatures ${ }^{2}$.

Due to the one-dimensional polymorphism, $\mathrm{SiC}$ exists in a large variety of crystal structures known as polytypes which correspond to a unique stacking sequence of $n$ successive $\mathrm{Si}-\mathrm{C}$ bilayers. In such arrangements every bilayer can be viewed as a two-dimensional hexagonal lattice of vertical $\mathrm{Si}-\mathrm{C}$ bonds. The large variety of polytypes implies a corresponding variety of properties such as the band gap energy. For example, SiC polytypes have a range of indirect band gaps ${ }^{3}$ from $2.39 \mathrm{eV}$ in the cubic polytype (3C) to $3.33 \mathrm{eV}$ in one of the hexagonal polytypes $(2 \mathrm{H})$. To date, the main focus of attention has been mainly drawn by $3 \mathrm{C}-\mathrm{SiC}^{4,5}$ and two hexagonal forms, $4 \mathrm{H}$ and $6 \mathrm{H}-\mathrm{SiC}^{6,7}$. In the case of $3 \mathrm{C}-\mathrm{SiC}$, this is because it has isotropic properties and it is the only SiC polytype that can be grown on a Si substrate.

Despite the progress made in $\mathrm{SiC}$ crystal growth techniques, the widespread application of SiC-based power devices is limited by the presence in both SiC bulk substrates and in the overgrown SiC epilayers of a high density of structural defects such as micropipes ${ }^{8}$, screw dislocations, basal plane dislocations and stacking faults ${ }^{9,10}$. In the case of 3C-SiC the most common defects are stacking faults (SFs), which are local regions of incorrect stacking of crystal planes, twin boundaries (TBs) which occur due to the twofold possibility to arrange the Si-C bilayer stacking along the c-axis on a (0001) hexagonal $\mathrm{SiC}$ substrate and double-positioning boundaries (DPBs) which are the dominant defects in $3 \mathrm{C}-\mathrm{SiC}$ films with (111) orientation grown on a hexagonal substrate and form due to a $60^{\circ}$ rotation of the initial $3 \mathrm{C}$ stacking on the $(0001)$ hexagonal plane ${ }^{11,12}$. It has been shown that such extended defects are detrimental to the behavior of semiconductor devices. The understanding of their origin is thus crucial for a broad range of $\mathrm{SiC}$-based applications and requires simple and readily available defect characterization methods for imaging defects present in the substrate or in the epilayer.

Various investigation techniques have been used to image and characterize different structural defects in SiC layers and devices, each technique having its own limitations. The optical beam induced current technique ${ }^{13}$, which is only suitable for device characterization was used to analyze n-type $4 \mathrm{H}$-SiC nickel Schottky barrier diodes. The imaging of extended SFs in degraded $\mathrm{SiC} \mathrm{PiN} \mathrm{diodes}{ }^{14}$ and the mapping of different defects in $\mathrm{SiC}^{15}$ was done by synchrotron white-beam X-ray topography. Although it is nondestructive in nature, the requirement 
of a synchrotron light source limits its use for routine characterization. X-ray diffraction (XRD) can yield information on polytypes and dislocations through indexing and peak-broadening analysis ${ }^{16}$, but achieving a spatial mapping capability is time-consuming. Transmission electron microscopy (TEM) can be used for the identification and evolution analysis of extended defects in 3C-SiC films $s^{17,18}$, but requires destructive sample preparation and severely limits the area of analysis to tens of microns or less. Scanning tunneling microscopy and atomic force microscopy (AFM) revealed atomic-scale images of $3 \mathrm{C}-\mathrm{SiC}$ and structural defects of the films, respectively ${ }^{19}$, but are limited to surface studies. Among the investigation techniques used for defect characterization in $\mathrm{SiC}$, nondestructive luminescence-based techniques helped to understand how defects in $\mathrm{SiC}$ are formed. The photoluminescence intensity mapping was used to identify three kinds of SFs in $4 \mathrm{H}-\mathrm{SiC}$ epilayers ${ }^{20}$, while cathodoluminescence and lifetime mapping were used to identify the stressed diodes after extended forward current operation ${ }^{21}$. Electroluminescence was used for imaging luminescent defects in PiN diodes in situ, during forward biasing, and ex situ, after degradation of the diodes ${ }^{22,23}$. Another optical technique, second harmonic generation (SHG) is known to be a rapid, noninvasive and sensitive investigation method used to identify different polytypes ${ }^{24}$ and structural defects in $\mathrm{SiC}$ epilayers ${ }^{25}$, to map microcrystalline inclusions of $\mathrm{SiC}$ polytypes in $6 \mathrm{H}-\mathrm{SiC}$ epilayers ${ }^{26}$ and to probe the crystalline order of $3 \mathrm{C}$-SiC films grown on (111) $\mathrm{Si}$ substrates by rotational anisotropy measurements ${ }^{27}$.

In the present work we propose SHG microscopy for the fast detection and identification of defects in $\mathrm{SiC}$ epilayers grown on hexagonal silicon carbide by the vapour-liquid-solid technique. By combining the SHG-based imaging with XRD and SHG rotational anisotropy the growth of $3 \mathrm{C}$ polytype on the $4 \mathrm{H}-\mathrm{SiC}$ substrate was confirmed and the polytype of the imaged defects was identified. If in previous publications ${ }^{24-27}$ optical SHG and especially SHG rotational anisotropy were used to detect polytype inclusions, identify different $\mathrm{SiC}$ polytypes, or determine crystalline properties for SiC film surfaces, in the present work we emphasize on its potential in high resolution imaging and in structural defect identification. In the same time, because defects detection and identification in $\mathrm{SiC}$ require high resolution surface characterization, AFM combined with SHG microscopy were employed so as to provide the topography of the samples needed to locate the defects imaged by SHG.

\section{Results}

$\mathrm{XRD}$ has been used to confirm the crystallinity and epitaxial quality of the $\mathrm{SiC}$ film (Fig. 1). The structure of the film was $3 \mathrm{C}-\mathrm{SiC}$ as indicated by strong cubic (111) and (222) diffraction peaks at $35.6^{\circ}$ and $75.3^{\circ}$ respectively, confirming the growth of the $3 \mathrm{C}-\mathrm{SiC}$ polytype. Since in the Bragg-Brentano configuration the diffraction data collected at each angle corresponds only to the structural planes parallel to the sample surface ${ }^{28}$, fig. 1 confirms that (111) $3 \mathrm{C}-\mathrm{SiC}$ is parallel with the surface and hence with the (0001) $4 \mathrm{H}-\mathrm{SiC}$ substrate on which the $3 \mathrm{C}-\mathrm{SiC}$ was grown. It can thus be concluded that the 3C-SiC layer was grown epitaxially with preferred orientation and highly aligned with the substrate. This is actually consistent with the two-dimensional nucleation mechanism for the $3 \mathrm{C}$ polytype growth.

In order to evaluate the potential of SHG-based microscopy to image defects in $\mathrm{SiC}$ epilayers we simultaneously collected confocal reflection (Fig. 2a) and SHG images (Fig. 2b) from a 3C-SiC film. Fig. 2 b displays a $x y$ scan of a $250 \times 250 \mu \mathrm{m}^{2}$ surface area, which contains an unusual number of defects; this region was intentionally selected to show different defects in a single SHG image. Visible defects, which can be seen in the reflection image often indicate but do not guarantee the presence of structural defects in the epilayer, making the detection of all defects in the epilayer nearly impossible. SHG imaging reveals elements that cannot be distinguished in the

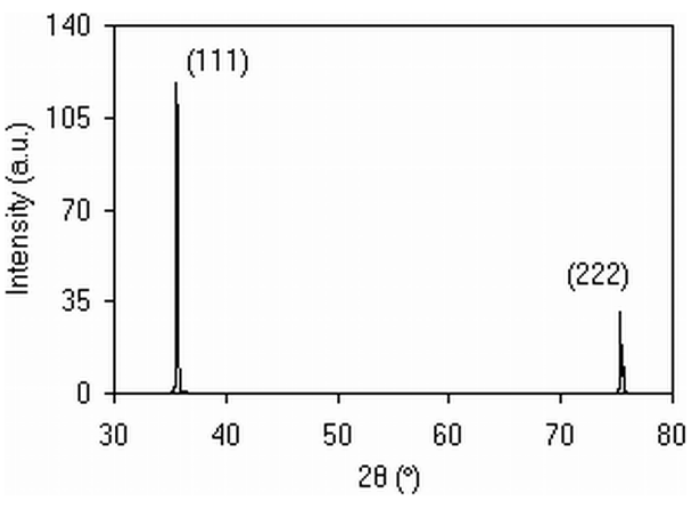

Figure $1 \mid \mathrm{X}$-ray diffractogram for the $3 \mathrm{C}$-SiC epilayer. The labeled peaks correspond to the cubic $\mathrm{SiC}$ crystal structure. No other foreign polytype-related reflection was observed in the XRD scans.

reflection image, which demonstrates the complementarity of the two imaging methods and propose SHG microscopy as a suitable technique to image not only superficial, but also buried structural defects.

The potential of SHG microscopy stretches beyond simple imaging as the polarization of the SHG radiation can be exploited to obtain additional information about the crystalline structure of the sample $^{26}$. In this regard, we have used SHG rotational anisotropy to identify the polytype for both defects and $\mathrm{SiC}$ epilayer. To determine the second harmonic ( $\mathrm{SH}$ ) rotational anisotropy of the samples illustrated in Fig. 2, $x y$ scans were performed at different polarization angles for the incident laser (Fig. 3).

In fig. $3 \mathrm{c}$ a dominant fourfold rotational symmetry and an additional twofold symmetry are visible. In the case of bulk 3C-SiC, a twofold rotational symmetry appears in components of the nonlinear susceptibility tensor. According to Neumann's principle, this twofold symmetry shows up also in the SH electric field, resulting in a fourfold symmetry in the SHG intensity ${ }^{24}$. The $3 \mathrm{C}$-SiC surface possesses a fourfold rotational crystal symmetry, which cannot be displayed in the $\mathrm{SH}$ rotational anisotropy due to the fact that the susceptibility tensor, being a third rank tensor, can only resolve symmetries up to threefold ${ }^{26}$. Thus, the contributions from the surface to the SHG intensity are purely isotropic when changing the laser polarization. The case of hexagonal $\mathrm{SiC}$ should also be taken into consideration because the $3 \mathrm{C}-\mathrm{SiC}$ which is of interest in our case is grown on a $4 \mathrm{H}-\mathrm{SiC}$ substrate. The surfaces of the hexagonal $\mathrm{SiC}$ polytypes that are cut parallel to the hexagonal $\mathrm{SiC}$ double layers, possess an overall sixfold symmetry which again gives rise only to an isotropic SH response. Only in the case of a misorientation of the surface away from the hexagonal planes an additional onefold contribution from the hexagonal $\mathrm{SiC}$ substrate will be introduced in the SH response.

In our case, the dominant fourfold symmetry confirms the presence of $3 \mathrm{C}-\mathrm{SiC}$ on the sample, while the twofold symmetry is originating from an isotropic contribution either from the $3 \mathrm{C}$-SiC surface or $4 \mathrm{H}-\mathrm{SiC}$ substrate. The fourfold symmetry dominates the twofold symmetry, because bulk contributions tend to dominate the surface contributions due to the longer interaction length in the case of bulk when compared to surfaces consisting of only a few atomic layers ${ }^{26}$.

Both defects and the epilayer have the same fourfold symmetry, with the intensity of the epilayer ten times lower compared to the case of defects. This difference in intensity is an advantage that can be used when imaging a $\mathrm{SiC}$ sample to identify defects from the lowerintensity epilayer.

The differences between the experimental data and the theory may be explained by quantitatively analyzing the case when the laser polarization is fixed (Fig. 4). The $\mathrm{SH}$ radiation is expected to have the polarization parallel with that of the incident laser beam and 

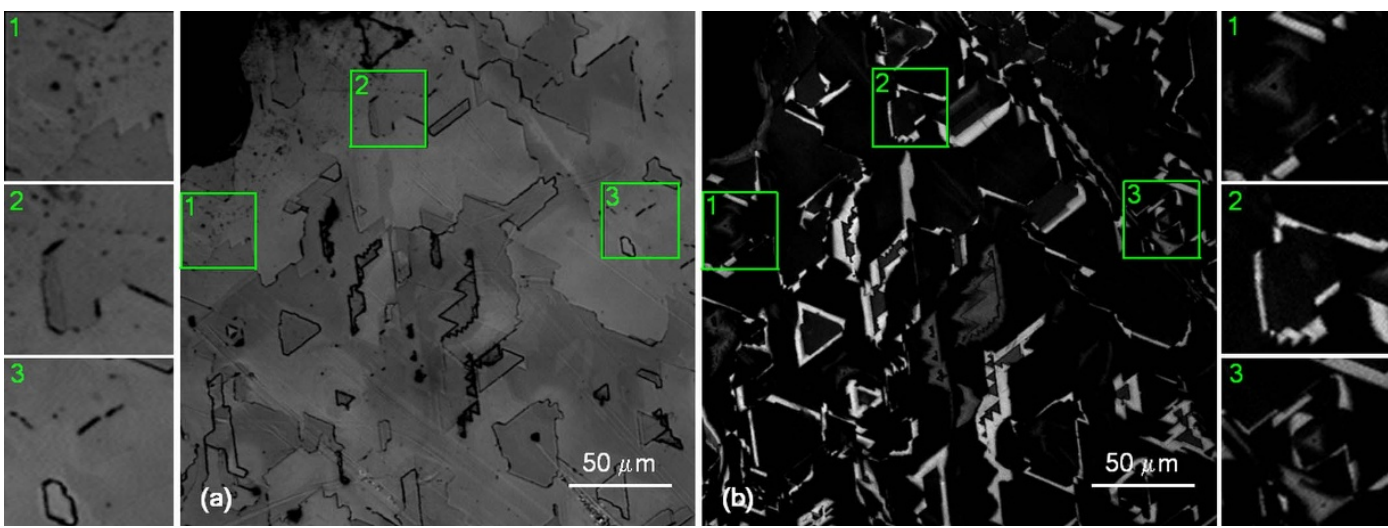

Figure $2 \mid$ Laser scanning microscopy images of 3C-SiC epilayer. (a) confocal reflection image; (b) SHG image. Both images are simultaneously collected from the same surface region. The three insets for both confocal and SHG images confirm that a surface inspection using confocal microscopy alone is not enough because some defects revealed in the SHG image cannot be observed in the reflection image.

therefore by rotating the analyzer before the microscope detector a $\cos ^{2} \theta$ pattern should result. Successive SHG images of the SiC sample were recorded rotating the analyzer by $360^{\circ}$ with $10^{\circ}$ steps. SHG intensity as a function of the analyzer rotation angle $(\theta)$ is well

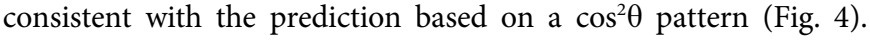
The deviation of the experimental data from the theoretical curve can be explained by the depolarization effects of galvanometric mirrors, the imaging objective and the transmission through the sample.

To further investigate the structure of the defects in the $\mathrm{SiC}$ films, SHG images and AFM topography in tapping mode were collected for the same areas on the sample surface (Fig. 5). Usually, morphological defects extend deep in the epilayer but form characteristic features on the surface. These surface features with distinct shapes can be easily identified when using optical microscopy imaging and can be linked with the corresponding defects that are buried below the sample surface. Triangular features are clearly discernible by confocal microscopy (Fig. 5a,d) and AFM (Fig. 5c,f), while SHG microscopy offers more details about the defects generating these features (Fig. 5b,e). SHG images confirm the extension of the surface features into the epilayer. Triangular features in the confocal image
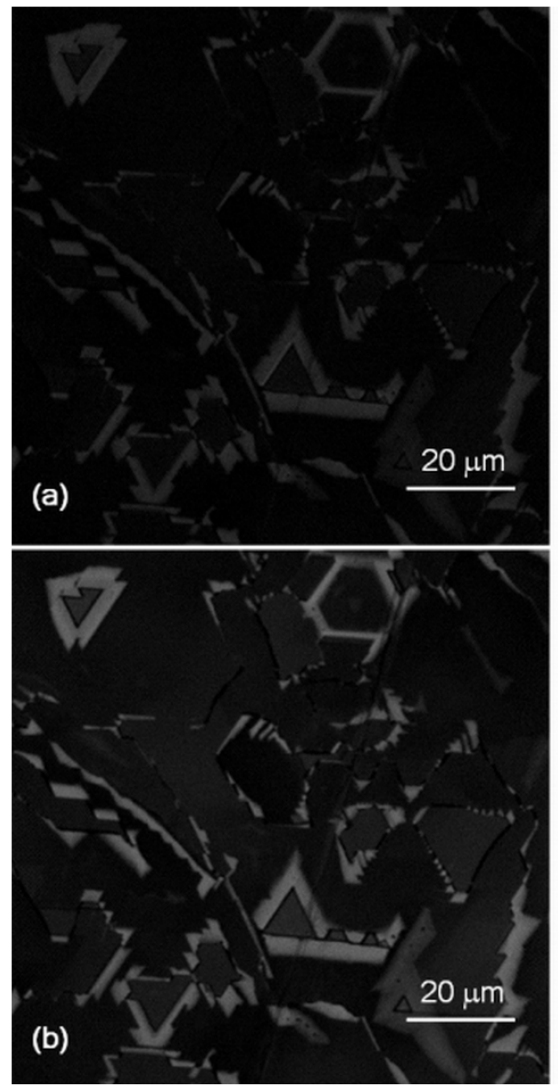

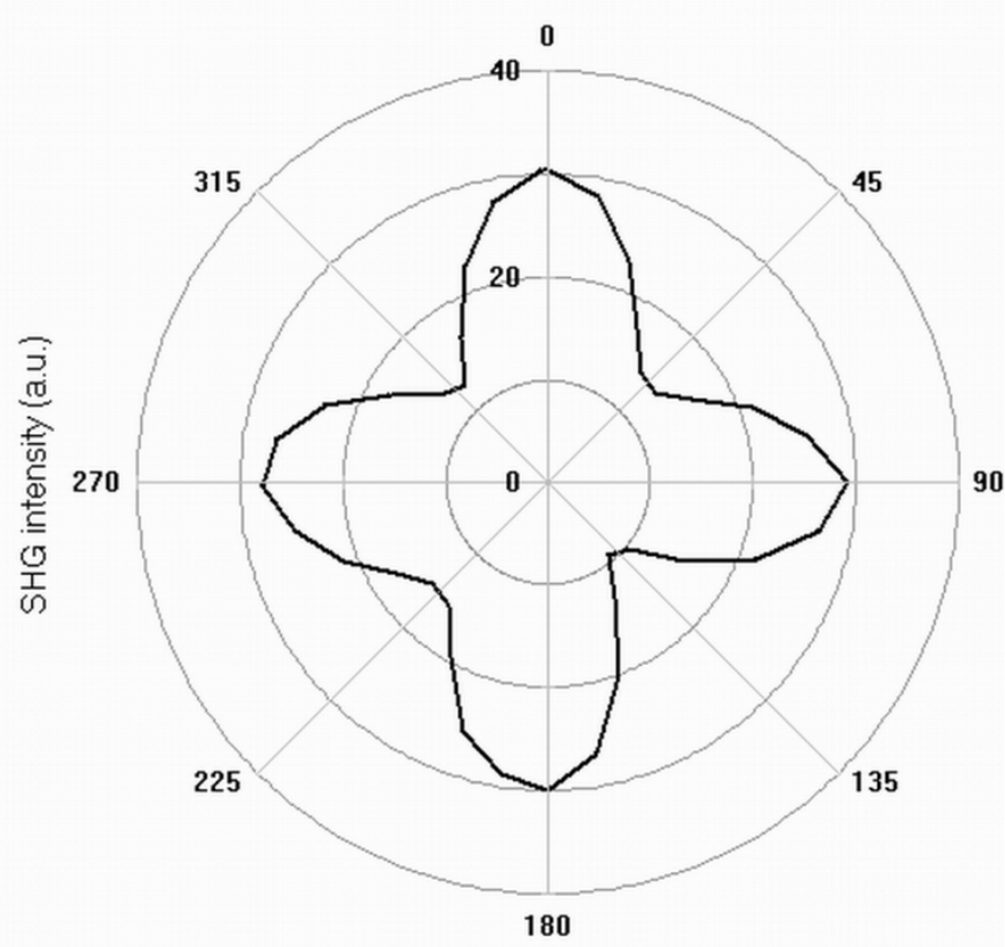

(c)

Azimuthal angle $\left(^{\circ}\right)$

Figure $3 \mid$ SH rotational anisotropy. By changing the polarization of the incident laser radiation, different SHG intensities are obtained. The corresponding SHG images at different polarization angles are displayed: (a) $50^{\circ}$; (b) $100^{\circ}$. The SHG intensity plot (c) is valid for both the epilayer and the defects. The plot for the epilayer intensity dependence with azimuthal angle is not shown due to lower intensity compared with that of the defects. The average SHG intensity from the defects is $\sim 10$ times higher than the SHG intensity from the SiC epilayer. Having the same rotational symmetry, both epilayer and defects are identified as 3C-SiC. 


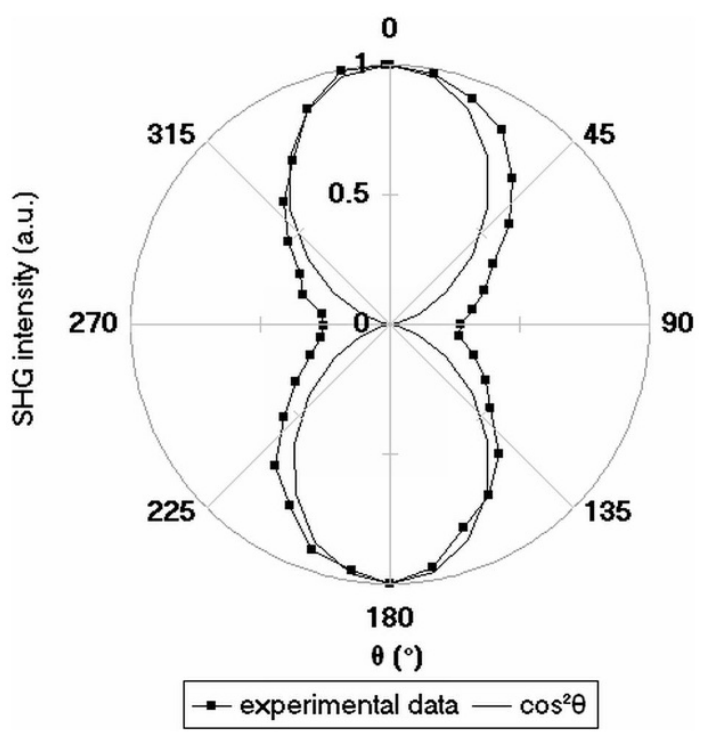

Figure $4 \mid$ The dependence of the SHG intensity on the rotation angle of the analyzer. By rotating the analyzer before the detector with $10^{\circ}$ steps, different intensities for the SHG are obtained. The experimental data fits well with the theoretical $\cos ^{2} \theta$ function.

(Fig. 5a) prove to be hexagonal in the SHG image with a buried central defect originating from DPBs (Fig. 5b). The distinct equilateral triangles are suspected to arise from partial dislocations bounding triangular stacking faults that have been previously resolved by using AFM on as-grown $3 \mathrm{C}$-SiC films ${ }^{29}$ and by using electron channeling contrast imaging ${ }^{30}$. Another source of the triangular features can be the existence of DPBs which preferentially expand along the (111) planes. Since the inclined (111) planes intersect the top (111) surface along $<110\rangle$ directions, the defects appear as equilateral triangles at the surface (Fig. 5) as also shown previously ${ }^{31}$.
Other defects visible by using SHG microscopy were also hexagonal-shaped. The size of such defects exceeds the usual AFM fieldof-view dimensions and were only investigated using SHG (Fig. 6). The hexagonal defects visible in the SHG image are DPBs which appear due to the growth of the $3 \mathrm{C}$ polytype on a hexagonal substrate. Similar hexagonal features were observed under an optical microscope $\mathrm{e}^{32}$ after potassium hydroxide $(\mathrm{KOH})$ etching of the $\mathrm{SiC}$ sample.

\section{Discussion}

The difference between the lattice parameters of the film and those of the substrate produces a lattice mismatch strain in the epilayer, with high influence near the film surface when imaging films of reduced thickness. This relative displacement of the elements causes local stress in the crystal lattice, that along with dislocations and stacking faults produces micro-strain across the film thickness, resulting in strain-induced SHG.

The presented experiment shows that SHG can be regarded as a viable characterization tool of $3 \mathrm{C}$-SiC layers. The ten times difference in SHG intensity between defects and background allows their rapid identification and a fast data acquisition, rotation of the sample being no longer necessary for finding the best sample position for optimal SHG intensity. SHG enables determining the SiC polytype by rotational anisotropy studies that can be performed by rotating the polarization angle of the incident laser. Full $\mathrm{SiC}$ wafers can also be imaged even with a relatively reduced field-of-view, by using automated stitching algorithms ${ }^{33}$ to create an image of the entire wafer after acquiring several SHG images to cover the whole wafer.

The nondestructive defect mapping method based on the principle of optical second harmonic generation that we introduced enables the rapid and in-depth identification of structural defects such as stacking faults, dislocations and double positioning boundaries in SiC epilyers. A major advantage of the SHG-based defect assessment method is that it can be extended to characterize any structural defects in SiC epilayers of different polytypes. Unlike TEM, X-ray
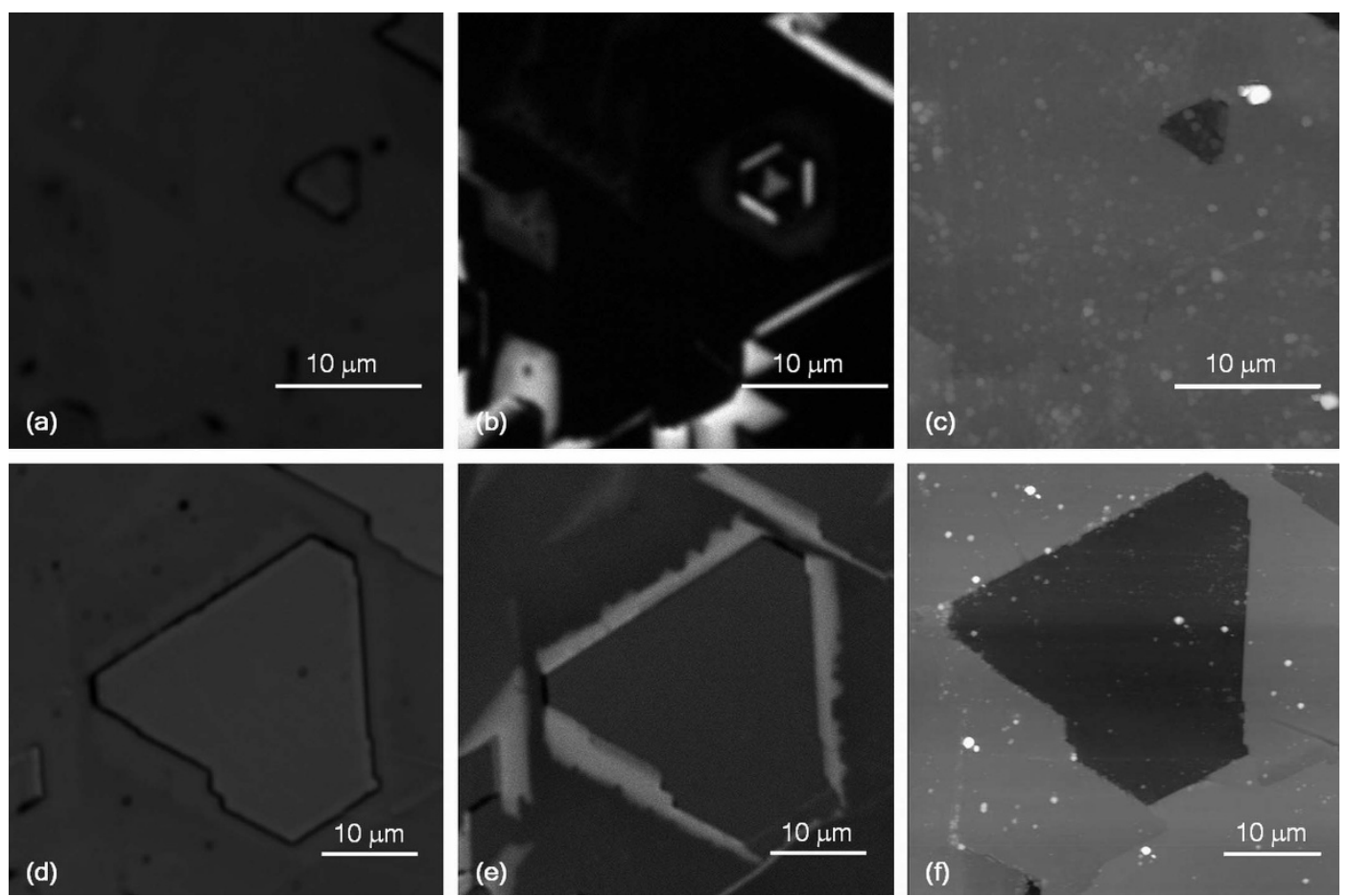

Figure 5 Combining AFM and laser scanning microscopy. Confocal (a), (d), SHG (b), (e) and AFM (c), (f) images of different defects in the 3C-SiC epilayer. Because the optical and AFM images are not perfectly registered but the scan area has the same size, confocal images are used as a reference to locate different surface features. Triangular features on the surface can be identified in SHG images as either DPBs (b) or triangular SFs (e). 


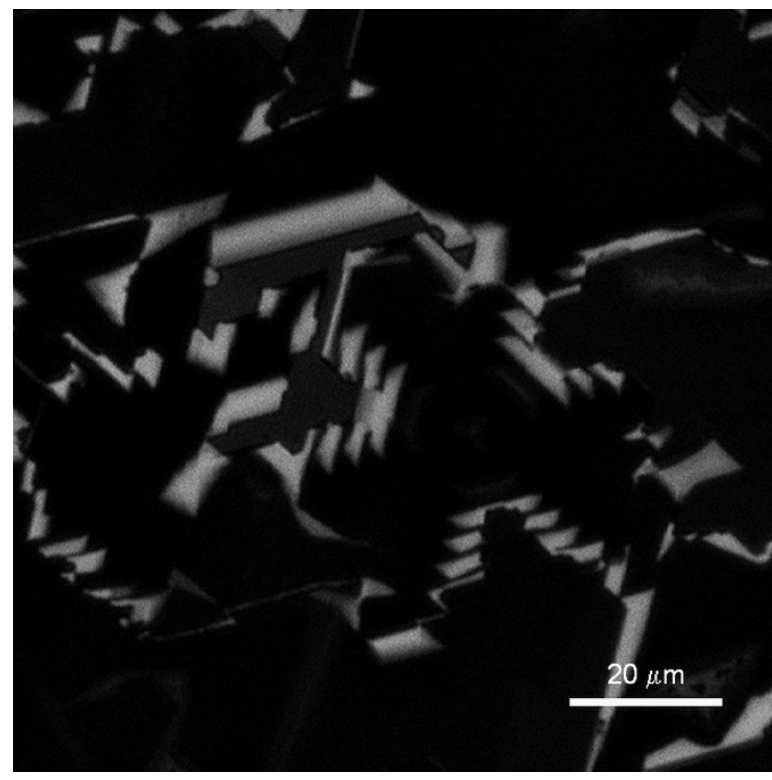

Figure 6 | Hexagonal defects in SHG microscopy. SHG image of hexagonal defects associated to DPBs which appeared due to the growth of cubic polytype on a hexagonal substrate.

topography or $\mathrm{KOH}$ etching, SHG-based techniques are nondestructive and require minimal sample preparation. Since the proposed SHG imaging technique is noninvasive and rapid, it can be used in situ in a production line to provide rapid feedback to processing engineers by highlighting areas within the $\mathrm{SiC}$ epilayer with structural defects. Such tasks could be performed also in automated scenarios, speeding the inspection process even more, by combining SHG microscopy with computer vision methods ${ }^{18}$ that can replicate analysis and inspection chores performed by human operators.

\section{Methods}

(111) 3C-SiC layers were grown by vapour-liquid-solid mechanism ${ }^{12,34}$ in a homemade vertical cold-wall quartz reactor using silicon-gallium (Si-Ga) melts on commercial on-axis (0001) $4 \mathrm{H}-\mathrm{SiC}$ wafers. The surface pretreatment included a slight hydrogen etching at $1450^{\circ} \mathrm{C}$ followed by a deposition of a Si layer on the seed at $1000^{\circ} \mathrm{C}$. A 1:3 Si: Ga composition was used for the melt, adjusting the growth temperature just above the melting point of the Si-Ga alloy at $1200^{\circ} \mathrm{C}$, in order to limit $\mathrm{Ga}$ loss by evaporation. The growth time was 30 minutes, resulting a film with a thickness of $\sim 900 \mathrm{~nm}$. More details about the fabrication procedure are given in previous publications $\mathrm{s}^{35,36}$

The XRD investigations were performed with a APD2000 (GNR Analytical Instruments Group, Italy) diffractometer in a Bragg-Brentano configuration.

SHG imaging was carried out by using a $50 \mathrm{~mW}$ laser beam, with $\sim 80 \mathrm{fs}$ pulses at $790 \mathrm{~nm}$ provided by a Spectra-Physics (now Newport Corporation, USA) Ti:Sapphire laser with a pulse repetition rate of $80 \mathrm{MHz}$. A Leica TCS SP confocal laser scanning microscope with a $40 \times, 0.7$ NA objective, was used to simultaneously acquire both confocal reflection and SHG images in a transmission configuration. A bandpass filter with the central wavelength at $390 \mathrm{~nm}$ (FB390-10, Thorlabs) placed before the detector ensured that only the SHG signal was detected. The SHG rotational anisotropy was measured by obtaining images through the rotation of an achromatic half-wave plate (AHWP05M-980, Thorlabs) placed before the microscope while maintaining a fixed position for the analyzer in front of the detector. Because both $3 \mathrm{C}$ - and $4 \mathrm{H}-\mathrm{SiC}$ are wide band gap materials, with $2.39 \mathrm{eV}$ and $3.26 \mathrm{eV}$ band gap energies, the wavelength used in our experiment $(790 \mathrm{~nm})$ and the corresponding wavelength of the SHG (395 nm) allowed for investigation in the volume of the sample and in a transmission configuration because no absorbtion of either the incident beam, nor the SHG ocurred in the sample.

A Q-Scope 350 AFM (Quesant, USA) was used for AFM imaging in tapping mode.

1. Shenai, K., Scott, R. S. \& Baliga, B. J. Optimum semiconductors for high-power electronics. IEEE T. Electron Devices 36, 1811-1823 (1989).

2. Niedermeier, S., Schillinger, H., Sauerbrey, R., Adolph, B. \& Bechstedt, F. Secondharmonic generation in silicon carbide polytypes. Appl. Phys. Lett. 75, 618-620 (1999).

3. Wu, I. J. \& Guo, G. Y. Second-harmonic generation and linear electro-optical coefficients of SiC polytypes and nanotubes. Phys. Rev. B: Solid State 78, 035447 (2008).
4. Severino, A. \& La Via, F. Microtwin reduction in 3C-SiC heteroepitaxy. Appl. Phys. Lett. 97, 181916 (2010).

5. Vasiliauskas, R., Juillaguet, S., Syvajarvi, M. \& Yakimova, R. Cubic SiC formation on the C-face of 6H-SiC (0001) substrates. J. Cryst. Growth 348, 91-96 (2012).

6. Twigg, M. E. et al. Structure of stacking faults formed during the forward bias of 4H-SiC p-i-n diodes. Appl. Phys. Lett. 82, 2410-2412 (2003).

7. Zhao, F., Islam, M. M., Daas, B. K. \& Sudarshan, T. S. Effect of crystallographic dislocations on the reverse performance of $4 \mathrm{H}-\mathrm{SiC}$ p-n diodes. Mater. Lett. 64, 281-283 (2010).

8. Wahab, Q. et al. Influence of epitaxial growth and substrate-induced defects on the breakdown of 4H-SiC Schottky diodes. Appl. Phys. Lett. 76, 2725-2727 (2000)

9. Kojima, K. et al. Influence of stacking faults on the performance of $4 \mathrm{H}-\mathrm{SiC}$ Schottky barrier diodes fabricated on (112̄0) face. Appl. Phys. Lett. 81, 2974-2976 (2002).

10. Fujiwara, H., Kimoto, T., Tojo, T. \& Matsunami, H. Characterization of in-grown stacking faults in $4 \mathrm{H}-\mathrm{SiC}(0001)$ epitaxial layers and its impacts on high-voltage Schottky barrier diodes. Appl. Phys. Lett. 87, 051912 (2005).

11. Kusumori, T., Muto, H., Okada, M. \& Jin, P. Reduction in double-positioning boundaries in $3 \mathrm{C}-\mathrm{SiC}$ epitaxial films fabricated on $\mathrm{Si}(111)$ substrates. Thin Solid Films 513, 307-310 (2006).

12. Soueidan, M. \& Ferro, G. A vapor-liquid-solid mechanism for growing $3 \mathrm{C}-\mathrm{SiC}$ single-domain layers on 6H-SiC(0001). Adv. Funct. Mater. 16, 975-979 (2006).

13. Tsuji, T. et al. Analysis of high leakage currents in $4 \mathrm{H}-\mathrm{SiC}$ Schottky barrier diodes using optical beam-induced current measurements. Mater. Sci. Forum. 389-3, 1141-1144 (2002).

14. Jacobson, H. et al. Properties and origins of different stacking faults that cause degradation in SiC PiN diodes. J. Appl. Phys. 95, 1485-1488 (2004).

15. Dudley, M., Huang, X. R. \& Vetter, W. M. Contribution of x-ray topography and high-resolution diffraction to the study of defects in SiC. J. Phys. D: Appl. Phys. 36, A30-A36 (2003)

16. Xie, Z. Y., Edgar, J. H., Burkland, B. K., George, J. T. \& Chaudhuri, J. DPBs-free and polytype controlled growth of $\mathrm{SiC}$ via surface etching on on-axis $6 \mathrm{H}$ $\mathrm{SiC}(0001)$. J. Cryst. Growth 224, 235-243 (2001).

17. Polychroniadis, E., Syvajarvi, M., Yakimova, R. \& Stoemenos, J. Microstructural characterization of very thick freestanding $3 \mathrm{C}-\mathrm{SiC}$ wafers. J. Cryst. Growth $\mathbf{2 6 3}$, 68-75 (2004)

18. Stanciu, S. G. et al. Automatic estimation of stacking fault density in $\mathrm{SiC}$ specimens imaged by transmission electron microscopy. Proceedings of the 13th International Conference on Transparent Optical Networks, Stockholm, Sweden, June 26-30 (2011).

19. Steckl, A. J., Roth, M. D., Powell, J. A. \& Larkin, D. J. Atomic probe microscopy of 3C SiC films grown on 6H SiC substrates. Appl. Phys. Lett. 62, 2545-2547 (1993).

20. Feng, G., Suda, J. \& Kimoto, T. Characterization of stacking faults in $4 \mathrm{H}-\mathrm{SiC}$ epilayers by room-temperature microphotoluminescence mapping. Appl. Phys. Lett. 92, 221906 (2008).

21. Sridhara, S. G., Carlsson, F. H. C., Bergman, J. P. \& Janzen, E. Luminescence from stacking faults in 4H SiC. Appl. Phys. Lett. 79, 3944-3946 (2001).

22. Galeckas, A., Linnros, J. \& Pirouz, P. Recombination-enhanced extension of stacking faults in $4 \mathrm{H}-\mathrm{SiC} \mathrm{p}-\mathrm{i}-\mathrm{n}$ diodes under forward bias. Appl. Phys. Lett. 81, 883-885 (2002).

23. Liu, K. X. et al. Photoluminescence and electroluminescence imaging of carrot defect in 4H-SiC epitaxy. J. Electron. Mater. 36, 297-306 (2007).

24. Jordan, C. et al. Characterization of silicon carbide surfaces of $6 \mathrm{H}-, 15 \mathrm{R}-$ and $3 \mathrm{C}-$ polytypes by optical second-harmonic generation in comparison with X-ray diffraction techniques. Appl. Phys. A: Mater. Sci. Process. 65, 251-257 (1997).

25. Hristu, R., Polychroniadis, E. K., Stanciu, S. G. \& Stanciu, G. A. Investigations on $\mathrm{SiC}$ by using nonlinear effects in scanning laser microscopy. Proceedings of the 13th International Conference on Transparent Optical Networks, Stockholm, Sweden, June 26-30 (2011)

26. Meyer, C., Lupke, G., Stein von Kamienski, E., Golz, A. \& Kurz, H. Nonlinear optical mapping of silicon carbide polytypes in 6H-SiC epilayers. Appl. Phys. Lett. 69, 2243-2245 (1996)

27. Galeckas, A., Petrauskas, M., Wahab, Q. \& Willander, M. Nonlinear optical investigation of silicon carbide surface properties. Nucl. Instrum. Methods Phys. Res. B 65, 357-360 (1992)

28. Kuzel, R. \& Bursik, J. On X-ray diffraction study of preferred grain orientations in polycrystalline thin films - Multicomponent texture in $\mathrm{KTaO} 3$ films. Thin Solid Films 530, 2-8 (2013)

29. Neudeck, P. G., Trunek, A. J. \& Powell, J. A. Atomic force microscope observation of growth and defects on as-grown (111) 3C-SiC mesa surfaces. Mater. Res. Soc. Symp. Proc. 815, 59-64 (2004).

30. Picard, Y. N. et al. Epitaxial SiC growth morphology and extended defects investigated by electron backscatter diffraction and electron channeling contrast imaging. J. Electron. Mater. 37, 691-698 (2008).

31. Speer, K. M., Neudeck, P. G., Crimp, M. A., Burda, C. \& Pirouz, P. Possible formation mechanisms for surface defects observed in heteroepitaxially grown 3C-SiC. Phys. Status Solidi A 204, 2216-2221 (2007).

32. Neudeck, P. G. et al. CVD growth of 3C-SiC on $4 \mathrm{H} / 6 \mathrm{H}$ mesas. Chem. Vap. Deposition 12, 531-540 (2006).

33. Stanciu, S. G., Hristu, R. \& Stanciu, G. A. Influence of confocal scanning laser microscopy specific acquisition parameters on the detection and matching of speeded-up robust features. Ultramicroscopy 111, 364-374 (2011). 
34. Andreadou, A. et al. Microstructural investigation of 3C-SiC islands grown by VLS mechanism on 6H-SiC substrate. J. Cryst. Growth 310, 1799-1803 (2008).

35. Marinova, M. et al. Influence of $\mathrm{Ga}$ doping on the microstructure of $3 \mathrm{C}-\mathrm{SiC}$ layers grown on $4 \mathrm{H}-\mathrm{SiC}$ substrates by VLS mechanism. Phys. Status Solidi C 10, 72-75 (2013).

36. Ferro, G. \& Jacquier, C. Growth by a vapour-liquid-solid mechanism: a new approach for silicon carbide epitaxy. New J. Chem. 28, 889-896 (2004).

\section{Acknowledgments}

The authors would like to thank Alkyoni Mantzari and Efstathios K. Polychroniadis from Physics Department, Aristotle University of Thessaloniki for providing the SiC samples. The presented work was partially supported by the UEFISCDI

PN-II-PT-PCCA-2011-3.2-1162 research grant, contract number 10/2012.

\section{Author contributions}

R.H. and G.A.S. conceived the experiments. R.H., S.G.S., D.E.T. and A.M. performed the experiments. G.A.S. coordinated the project. R.H., A.M. and G.A.S. wrote the paper. All authors contributed through scientific discussions and reviewed the manuscript.

\section{Additional information}

Competing financial interests: The authors declare no competing financial interests.

How to cite this article: Hristu, R., Stanciu, S.G., Tranca, D.E., Matei, A. \& Stanciu, G.A. Nonlinear optical imaging of defects in cubic silicon carbide epilayers. Sci. Rep. 4, 5258; DOI:10.1038/srep05258 (2014)

This work is licensed under a Creative Commons Attribution-NonCommercialNoDerivs 4.0 International License. The images or other third party material in this article are included in the article's Creative Commons license, unless indicated otherwise in the credit line; if the material is not included under the Creative Commons license, users will need to obtain permission from the license holder in order to reproduce the material. To view a copy of this license, visit http:// creativecommons.org/licenses/by-nc-nd/4.0/ 